\title{
Autologous Dendritic Cell-Autologous Tumor mRNA-Human CD40L Vaccine
}

National Cancer Institute

\section{Source}

National Cancer Institute. Autologous Dendritic Cell-Autologous Tumor mRNA-Human

CD40L Vaccine. NCI Thesaurus. Code C61077.

A cancer vaccine consisting of autologous dendritic cells transfected with autologous tumor mRNA and the human CD40 ligand (CD40L) gene with immunostimulatory and antitumor activities. Vaccination with autologous dendritic cell-autologous tumor mRNAhuman CD40L vaccine may elicit a cytotoxic T cell response against tumor cells from which the autologous tumor mRNA was derived. When expressed by dendritic cells, tumor antigens and the co-stimulatory molecule CD40L, which binds to CD40 receptors on antigen presenting cells (APC), facilitate both humoral and cellular immune responses against tumor cells. 